\title{
Magnetoelastic Effects and Random Magnetic Anisotropy in Highly Strained Ultrathin Ni Nanowires Epitaxied in a $\mathrm{SrTiO}_{3}$ Matrix
}

\author{
X. Weng ${ }^{\mathrm{a}}$, M. Hennes ${ }^{\mathrm{a}, 1}$, D. Hrabovsky ${ }^{\mathrm{b}}$, D. Demaille ${ }^{\mathrm{a}}$, F. Vidal ${ }^{\mathrm{a}}$, Y. Zheng ${ }^{\mathrm{a}}$ \\ ${ }^{a}$ Sorbonne Université, CNRS-UMR 7588, Institut des NanoSciences de Paris (INSP), F-75005 Paris, France \\ ${ }^{b}$ Sorbonne Université, Plateforme Mesures Physiques à Basses Températures (MPBT), F-75005 Paris, France
}

\begin{abstract}
We analyze the magnetic anisotropy of Ni nanowires with diameters smaller than $5 \mathrm{~nm}$. The nanowires are vertically epitaxied in a $\mathrm{SrTiO}_{3}(001)$ matrix which generates huge tensile strains up to $3.6 \%$ along the nanowire axis. This leads to an unusual anisotropy, characterized by an easy magnetization plane perpendicular to the nanowire axis. Hysteresis cycles $M(H)$ unveil an overall in-plane isotropy, while an opening of the $M(H)$ cycles and thermal activation measurements indicate the presence of local energy barriers inside the nanowires. Surprisingly, the coercive field $H_{c}(T)$ decays exponentially with increasing temperature, for both the easy plane and the hard axis. Based on these findings, we provide an analysis of magnetoelastic effects in the nanowires. By considering global averaging over the anisotropy distribution and local averaging according to the Random Magnetic Anisotropy model, we find that the global anisotropy, with its hard axis and isotropic easy plane, is related to the mean strain, while coercivity arises from local strain variations. We evidence that a thermally activated anisotropy softening occurs in the nanowires, in addition to Sharrock's law of thermal reduction of coercivity. Possible mechanisms responsible for this thermal softening of anisotropy are proposed and discussed. Our study eventually allows to identify two major competing effects at play in the present system: an increasing magnetic anisotropy with increasing strain and a reduction of the anisotropy with increasing local strain fluctuations.
\end{abstract}

Keywords:

Nickel nanowires, Magnetic anisotropoy, Strain, Magnetoelastic effect, Vertical epitaxy

PACS: 81.07.Gf, 75.80.+q, 75.30.Gw, 75.75.-c

\section{Introduction}

Vertically aligned nanocomposites (VANs), i.e. thin films composed of arrays of vertically epitaxied nanopillars, have recently emerged as a novel and promising category of materials [1-11]. Thanks to the large interface between the matrix and the nanopillars, and as a consequence of their epitaxial growth, coupling effects can occur, thereby enhancing the properties of the matrix and/or nanopillars. This paves the way for synthesis of multifunctional materials and offers exciting perspectives for applications in the fields of plasmonics [1-3], catalysis [4], ion conductivity [5], ferroelectricity [6], multiferroic materials [7], magnetotransport and spintronics $[8,9]$ and high density data storage $[10,11]$.

Epitaxial strain constitutes an efficient lever to tune the physical properties of VANs. For example, the in-plane strain in $(\mathrm{BaSr}) \mathrm{TiO}_{3}$ films can be varied by the content of vertical $\mathrm{Sm}_{2} \mathrm{O}_{3}$ nanopillars and enhanced ferroelectric properties were obtained compared to pure $(\mathrm{BaSr}) \mathrm{TiO}_{3}$ films [6]. In-plane strain was also exploited in self-assembled vertical $\left(\mathrm{BaTiO}_{3}-\mathrm{CoFe}_{2} \mathrm{O}_{4}\right.$ and $\left.\mathrm{PbTiO}_{3}-\mathrm{CoFe}_{2} \mathrm{O}_{4}\right)$ thin films [7]. Another approach is to take advantage of the vertical strain in nanopillars induced by the vertical epitaxy [12-14]. It was demonstrated that the strain

\footnotetext{
${ }^{1}$ M.H. presently at LCPMR, Sorbonne Université, CNRS-UMR 7614, F75005 Paris, France.
}

state of $\mathrm{CoFe}_{2} \mathrm{O}_{4}$ nanopillars and consequently their magnetic behavior can be modulated by changing the matrix among $\mathrm{BiFeO}_{3}, \mathrm{PbTiO}_{3}, \mathrm{SrTiO}_{3}$ and $\mathrm{SrRuO}_{3}$ [14]. Even larger strains can be achieved in metal/oxide systems, by exploiting the large lattice mismatch and using sequential growth schemes, resulting in metastable nanopillar states [15]. Considering the balance between the interface energy and the volume elastic energy, the vertical strain of the nanopillars should increase as their diameter decreases. We achieved this using ultrathin $\mathrm{Ni}$ nanowires (NWs) in a $\mathrm{SrTiO}_{3}$ (STO) matrix with a vertical lattice mismatch of $11 \%$ : the NW axial tensile strain is increased from 2 to about $4 \%$, when reducing the diameter of Ni NWs from 6 towards $2 \mathrm{~nm} \mathrm{[16].}$

Here, we analyse the magnetic behavior of these highly strained ultrathin Ni NWs. The paper is organized as follows. The measurement methods used in the present work will be given at first (§2). Morphological and structural key features of Ni NWs will be described, and special attention will be paid to the strain induced in NWs by vertical epitaxy (\$3.1). Results on the magnetic anisotropy of Ni NWs will be shown and the origin of local energy barriers analyzed (\$3.2). The thermal evolution of coercivity will be thoroughly investigated by measurements and simulation (\$3.3). We will discuss ( $\$ 4)$ and conclude (\$5) on the interplay between the strain and the magnetic anisotropy in ultrathin Ni NWs. 


\section{Experiments}

Ni NWs were grown by sequential pulsed laser deposition (PLD) in a STO matrix on STO(001) substrates, using procedures already described in previous works [17-19]. A quadrupled Nd:YAG laser (wavelength $266 \mathrm{~nm}$ ) operating at $10 \mathrm{~Hz}$ with a fluence in the $1-3 \mathrm{~J} . \mathrm{cm}^{-2}$ range was used. The pressure during growth was of the order of $10^{-8}$ mbar for sample A and $10^{-6}$ mbar for samples B and C (Tab.1). The temperature of the substrate was kept close to $650^{\circ} \mathrm{C}$. The growth of the vertically self-assembled STO-embedded Ni NWs was performed by shooting alternatively on $\mathrm{NiO}$ and STO targets. The diameter and density of Ni NWs was tuned by adjusting the number of laser shots on each target, as illustrated in Fig.1(a). Each basic sequence deposited an amount of the order of $0.1 \mathrm{~nm}$ of STO matrix and of $0.01 \mathrm{~nm}$ of $\mathrm{Ni}$, in terms of equivalent thickness of a continuous STO or Ni layer respectively.
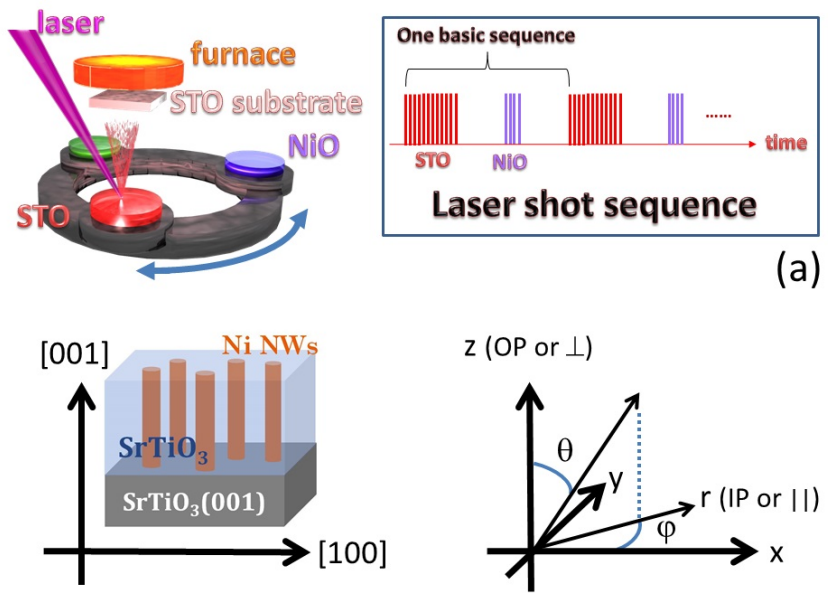

(b)

Figure 1: (a) Schematics of the sequential deposition process alternating laser ablation of STO and NiO targets. (b) Sketch of Ni NWs vertically epitaxied in a STO matrix on a STO $(001)$ substrate: the out-of-plane (OP or $\perp$ ) direction corresponds to the [001] or Z-direction; an in-plane direction (IP or \|) corresponds to one in the film plane.

The structure of the samples was studied using transmission electron microscopy (TEM) and x-ray diffraction (XRD). High resolution TEM (HRTEM) images were acquired using a JEOL JEM 2100F microscope equipped with a field-emission gun operated at $200 \mathrm{kV}$ and a Gatan GIF spectrometer. XRD data were collected on a laboratory 5-circle diffractometer (Rigaku SmartLab) with $\mathrm{Cu} \mathrm{K}_{\alpha}$ radiation. The $\mathrm{Ni} \mathrm{NW}$ axial strain was measured by XRD 2 $\theta / \omega$ scans and out-of-plane (OP) reciprocal space mapping (RSM). In the OP geometry, $\mathrm{Cu} \mathrm{K} \mathrm{K}_{\alpha 1}$ radiation was selected by a channel-cut $\mathrm{Ge}(220) 2$ reflexion monochromator. The radial strain was measured by in-plane (IP) $2 \theta_{\chi} / \varphi$ scans and IP-RSM at the grazing incidence of $0.5^{\circ}$ without the monochromator. Furthermore, a series of similar samples was analyzed by XRD at the SixS beamline and X-ray absorption spectroscopy at Ni K-edge at the SAMBA beamline of Synchrotron SOLEIL [16].
Magnetic measurements were performed using a vibrating sample magnetometer (VSM) option in a physical properties measurement system (Quantum Design PPMS 9T) and a superconducting quantum interference device (SQUID) magnetometer (Quantum Design MPMS XL). The longitudinal magnetization $(M)$ was measured as a function of the magnetic field $(H)$ in OP and IP geometry (Fig.1(b)). The magnetic hysteresis cycles were corrected by removing the diamagnetic signal (linear slope of the curves at large magnetic fields) in order to extract the ferromagnetic part corresponding to the response of the NW assembly. First-order reversal curves (FORCs) were collected according to the protocol of Pike et al. [20] and processed with the FORCinel software [21]. Magnetization measurements as a function of the temperature were also performed using field cooling (FC) and zero field cooling (ZFC) protocols.

\section{Results and analysis}

\subsection{Structural features}

The structural characteristics of ultrathin Ni NWs were detailed previously [16]. The main features of the present samples are summarized in Table 1. A TEM plan view of Ni NWs in sample $\mathrm{C}$ is presented in Fig.2(a) and the cube-on-cube epitaxy relationship can be seen in a HRTEM plan view (Fig.2(b)) where the alignement of $\mathrm{Ni}$ and STO crystalline axes becomes apparent. Complementary cross-sectional TEM observation indicates that NWs go through the whole thickness of the film. A narrow distribution of NW diameters was found for all the samples with a relative deviation from the mean value in the range of $10-16 \%$.

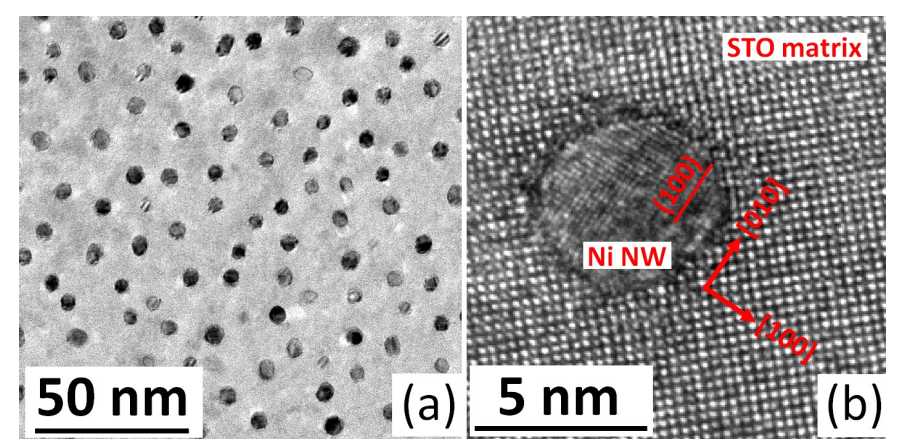

Figure 2: (a) TEM plan views of Ni NWs in sample C, with (b) a detailed view on one NW showing the alignment of the lattice planes of $\mathrm{Ni}$ with that of the surrounding STO matrix.

XRD measurements confirmed the epitaxy of Ni NWs in the STO matrix on a STO(001) substrate, as illustrated by the OP and IP RSMs around Ni and STO 002 and 200 spots, respectively (Fig.3). One can notice that the epitaxy of the STO matrix on the substrate is pseudomorphic, since the IP matrix spot 200 overlaps that of the substrate (Fig.3(b)). The Bragg angle of the matrix spot 002 is smaller than that of the substrate which indicates that the matrix OP lattice parameter increases from the bulk value $(0.3905 \mathrm{~nm})$ to the range of $0.393-0.398 \mathrm{~nm}$ (Fig.3(a)). We stress that the vertical lattice mismatch between 
Table 1: Structural characteristics of samples A-C determined by TEM and XRD measurements: mean value $\langle D\rangle$ and standard deviation $\sigma(D)$ of the diameter distribution; $\left\langle\epsilon_{z z}\right\rangle$ and $\sigma\left(\epsilon_{z z}\right)$ of the Ni NW axial strain; mean radial strain $\left\langle\epsilon_{r r}\right\rangle ; P$ : porosity, i.e. Ni volume fraction; $t$ : film thickness.

\begin{tabular}{cccccccc}
\hline & $\langle D\rangle$ & $\sigma(D)$ & $\left\langle\epsilon_{z z}\right\rangle$ & $\sigma\left(\epsilon_{z z}\right)$ & $\left\langle\epsilon_{r r}\right\rangle$ & $P$ & $t$ \\
& $(\mathrm{~nm})$ & $(\mathrm{nm})$ & $(\%)$ & $(\%)$ & $(\%)$ & & $(\mathrm{nm})$ \\
\hline $\mathrm{A}$ & 2.5 & 0.4 & 3.6 & 1.6 & -1.0 & 0.19 & 77 \\
$\mathrm{~B}$ & 4.1 & 0.5 & 3.0 & 1.5 & -0.4 & 0.24 & 231 \\
$\mathrm{C}$ & 5.0 & 0.5 & 2.4 & 1.3 & -0.3 & 0.10 & 106 \\
\hline
\end{tabular}

$\mathrm{Ni}$ and STO is as high as $11 \%$. The vertical epitaxy of embedded Ni NWs leads to an OP NW lattice expansion $\left\langle\epsilon_{z z}\right\rangle$ up to $3.6 \%$ with respect to the bulk (Fig.3(a)) and leaves a remaining vertical lattice mismatch of $\sim 8 \%$. One remarkable fact is that the axial strain $\left\langle\epsilon_{z z}\right\rangle$ is increasing with decreasing NW diameter $\langle D\rangle$. The consequent IP lattice compression $\left\langle\epsilon_{r r}\right\rangle$ of Ni NWs is ranged from $-0.3 \%$ to $-1.0 \%$, which is significantly lower than $\left\langle\epsilon_{z z}\right\rangle$ in terms of absolute value (Fig.3(b); Tab.1). It should be underlined that the vertical strain $\left\langle\epsilon_{z z}\right\rangle$ obtained here is much higher than the reported values in other nanocomposites: for instance, $\leq 1.5 \%$ for $\mathrm{Ni} \mathrm{NWs}$ in $\mathrm{CeO}_{2}$ matrix [15], $\leq 2.0 \%$ for $\mathrm{MgO}$ and $\leq 0.5 \%$ for $\mathrm{ZnO}$ in $(\mathrm{LaSr}) \mathrm{MnO}_{3}$ [12], a compressive vertical strain of $-0.8 \%$ in $\mathrm{BaTiO}_{3}$ matrix [13] and $-1.2 \%$ in $\mathrm{SrRuO}_{3}$ [14] for $\mathrm{CoFe}_{2} \mathrm{O}_{4}$ nanopillars.

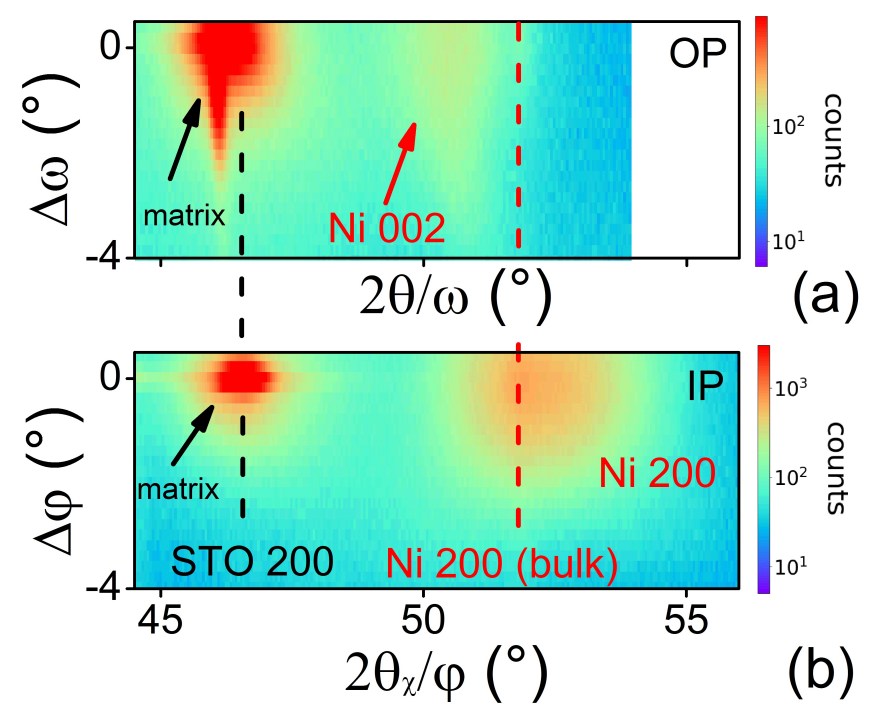

Figure 3: XRD RSMs of sample C: the Ni and STO bulk Bragg angles are indicated by the vertical red and black dashed lines, respectively; the tensile axial strain $\epsilon_{z z}$ and compressive radial strain $\epsilon_{r r}$ are measured by the departure of Ni NW Bragg angles from the bulk position. (a) OP RSM around Ni and STO 002 spots. (b) IP RSM around Ni and STO 200 spots.

One main characteristic feature is the wide spreading of $\mathrm{Ni}$ NW 002 and 200 spots in the RSMs. Detailed analysis of synchrotron XRD and XAS measurements [16] showed that NWs are composed of structurally coherent domains connected along the NW axis.

The coherence lengths are estimated to range from 6 to 13 $\mathrm{nm}$ in samples $\mathrm{B}$ and $\mathrm{C}$ and to lie around $6 \mathrm{~nm}$ in the thinnest and most strained sample A. Consequently, the OP profile of

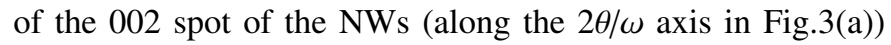
can be understood as the convolution of the axial strain $\epsilon_{z z}$ and the coherence length form factor. The axial strain $\epsilon_{z z}$ displays a large distribution with a deviation $\sigma\left(\epsilon_{z z}\right)$ of about $50 \%$ of $\left\langle\epsilon_{z z}\right\rangle$ (Tab.1). Furthermore, strain relaxation goes along with a 34 degrees angular tilting around the [100] or [010] axis [16]. This mosaicity is visualized in the OP-RSM by the spreading of the NW 002 spot along the sample rocking angle ( $\Delta \omega$ axis in Fig.3(a)). In the last case, the rocking profile also results from the convolution of the mosaicity and the NW diameter form factor. One important feature of strain relaxation is an increase in coherence length with decreasing strain. Concerning the distribution of the radial strain $\epsilon_{r r}$, few details were obtained because of the smallness of the NW diameters and low resolution of the IP x-ray optics used. Nevertheless, synchrotron XRD results obtained on sample B indicated that the deviation $\sigma\left(\epsilon_{r r}\right)$ is larger than $0.1 \%$.

We conclude from this structural analysis that Ni NWs are composed of vertical domains with coherence length smaller than $13 \mathrm{~nm}$ and with a highly dispersed strain between domains. As will be shown in the following, this peculiar NW structure and especially the broad strain distributions have an important impact on the magnetic behavior of the NWs.

\subsection{Origin of the magnetic anisotropy}

\subsubsection{Results at low temperature}

The main magnetic characteristics of the samples obtained at $10 \mathrm{~K}$ are listed in Table 2, together with the blocking temperatures $\left(T_{b}\right)$. OP and IP hysteresis cycles $M(H)$ of samples A and $\mathrm{B}$ at $10 \mathrm{~K}$ are displayed in Figure 4 . One can notice straightaway: a) the NW axis is the magnetization hard axis and the film plane the easy plane; b) the similarity of the cycles measured along the azimuthal [100] and [110] directions indicates the overall isotropy in the easy plane; c) an opening of the cycles occurs for both the hard axis and the easy plane.

Table 2: IP $(\|)$ and OP $(\perp)$ magnetic hysteresis characteristics of samples A-C. $T_{b}$ (blocking temperature) and values at $10 \mathrm{~K}: H_{c}$ (coercive field), $H_{s}$ (saturation field) and $m_{R}$ (remanence).

\begin{tabular}{c|ccccc}
\hline & & $\begin{array}{c}T_{b} \\
(\mathrm{~K})\end{array}$ & $\begin{array}{c}\mu_{o} H_{c} \\
(\mathrm{mT})\end{array}$ & $\begin{array}{c}\mu_{o} H_{s} \\
(\mathrm{~T})\end{array}$ & $m_{R}$ \\
\hline $\mathrm{A}$ & $\|$ & $26 \pm 3$ & $17 \pm 2$ & $0.48 \pm 0.07$ & $0.42 \pm 0.02$ \\
$\mathrm{~B}$ & $\|$ & $80 \pm 10$ & $47 \pm 2$ & $0.83 \pm 0.09$ & $0.54 \pm 0.02$ \\
$\mathrm{C}$ & $\|$ & $150 \pm 30$ & $71 \pm 2$ & $0.75 \pm 0.14$ & $0.61 \pm 0.03$ \\
\hline $\mathrm{A}$ & $\perp$ & $23 \pm 7$ & $17 \pm 5$ & $0.83 \pm 0.11$ & $0.13 \pm 0.03$ \\
$\mathrm{~B}$ & $\perp$ & $97 \pm 10$ & $69 \pm 2$ & $1.10 \pm 0.10$ & $0.36 \pm 0.02$ \\
$\mathrm{C}$ & $\perp$ & $200 \pm 30$ & $95 \pm 10$ & $0.85 \pm 0.10$ & $0.40 \pm 0.05$ \\
\hline
\end{tabular}

The IP isotropy is intriguing. It cannot be related to misoriented Ni crystalline axes, since the mosaicity of the epitaxied NWs is $\leq 4^{\circ}$, as established by our structural analysis. Experiments and calculations on ultrathin Ni layers [22, 23] show that a tetragonalization of the crystal goes along with a tetragonal 
magnetic symmetry. For the present case, only slight differences might be distinguished between the IP [100] and [110] directions when approaching saturation (Fig.4). This could be due to the measurement uncertainty. No feature from the observations can be unambiguously linked to the magnetocrystalline effect.

With increasing strain, the difference between the OP and IP cycles $M(H)$ is increased and the relative character of a hard axis reinforced. If we take the ratio $H_{s, \perp} / H_{s, \|}$ as a criterion, we observe that it increases from 1.1 to 1.7 , with $\left\langle\epsilon_{z z}\right\rangle$ varying from $2.4 \%$ (sample $\mathrm{C}$ ) to $3.6 \%$ (sample $\mathrm{A}$ ). This is also reflected by decreasing remanences $m_{R, \perp}$ with increasing strain. The saturation fields along the hard axis $\left(\mu_{o} H_{s, \perp}\right)$, between $0.83-1.10 \mathrm{~T}$, do not simply follow the mean strain.

Besides IP isotropy, another peculiar feature of the present system is its tendency to display similar IP and OP coercive fields. In both measurement configurations, $H_{c}$ decreases with increasing mean strain, which could however also be linked to the decrease in NW diameter. Finally, it should be noticed that $H_{c}$ is one order of magnitude smaller than $H_{s}$.
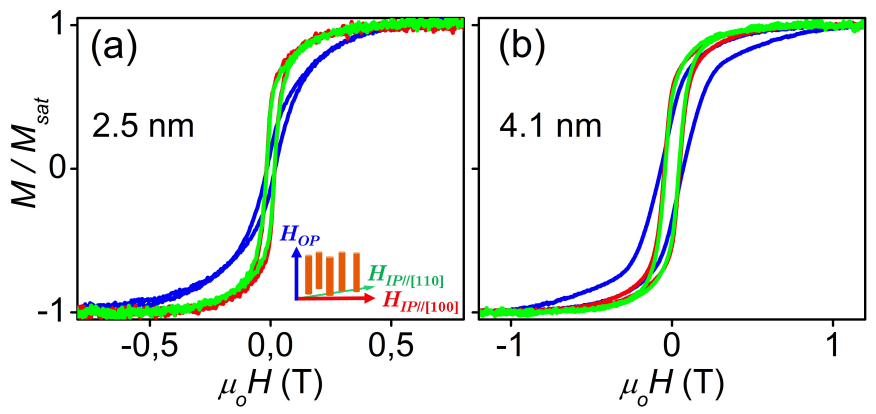

Figure 4: Hysteresis cycles measured at $10 \mathrm{~K}$ along the OP (blue), IP [100] (red) and IP [110] (green) directions in samples A (a) and B (b). Vertical scale: normalized magnetization.

In summary, we find that in the present system, the magnetic anisotropy is not dominated by the shape of the NWs, but by tensile strain, which reaches large values around 3\%. A first analysis of the data unravels a variety of pecularities and two main questions emerge: a) what is the origin of the IP and OP coercivities observed? b) why is the easy plane isotropic? For the analysis of the results, our approach will consist in identifying the local energy barriers responsible for the unexpected opening of the cycles as well as the corresponding activation volumes, in order to understand the main magnetic characteristics of this composite system.

\subsubsection{Anisotropy within the Stoner-Wohlfarth model}

We start this section by providing theoretical predictions based on the Stoner-Wohlfarth (SW) model [24] for entirely coherent magnetization reversal in Ni NWs. Due to the high length/diameter aspect ratio, the shape effect leads to an easy axis along the NWs which can be described by the uniaxial magnetostatic anisotropy constant: $K_{m s}=\left(\mu_{o} / 4\right) M_{s}^{2}$ with $M_{s}$ the saturation magnetization density $\left(5.1 \times 10^{5} \mathrm{~A} / \mathrm{m}\right.$ at $0 \mathrm{~K}$ [25]). $K_{m s}$ is equal to $0.8 \times 10^{5} \mathrm{~J} . \mathrm{m}^{-3}$ for Ni. Tensile strain in a Ni NW plays against the shape effect, because of the negative magnetostriction constant $\lambda_{001}\left(-5.5 \times 10^{-5}\right.$ [26]). The magnetoelastic anisotropy constant $K_{m e}$ is then: $K_{m e}=(3 / 2) \lambda_{001}\left(c_{11}-c_{12}\right)\left(\epsilon_{z z}-\right.$ $\left.\epsilon_{r r}\right)$ where $c_{i j}$ are the elastic constants. Compensation between the two effects occurs for a strain $\left(\epsilon_{z z}-\epsilon_{r r}\right)$ of $0.8 \%$. For a strain of about $3 \%$, the total constant $K_{m e}+K_{m s}$ is around $-2.0 \times 10^{5}$ $\mathrm{J}^{\mathrm{m}} \mathrm{m}^{-3}$. Thus, for strains exceeding the critical value of $0.8 \%$, we expect an anisotropic behavior characterized by a hard axis and an easy plane, without any coercivity (Fig.5(a)).

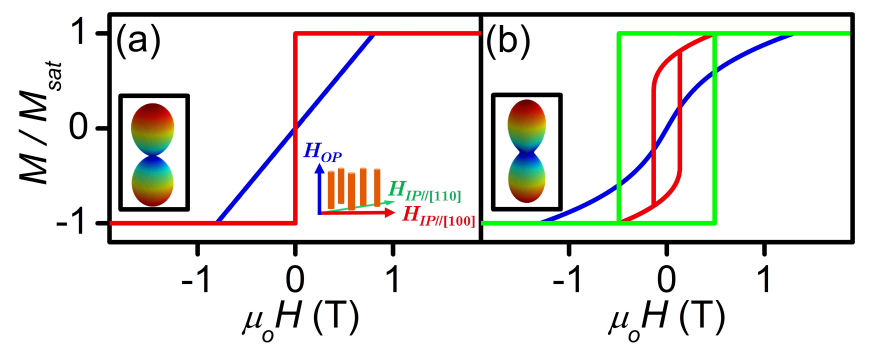

Figure 5: Relative magnetization of a Ni NW according to the Stoner-Wohlfarth model for a strain $\left(\epsilon_{z z}-\epsilon_{r r}\right)$ of $3 \%$. (a) Uniaxial case with $K_{m e}$ and $K_{m s}$ : OP (blue) and IP (red) curves; insert: corresponding anisotropy energy $\mathrm{E}(\theta, \varphi)$ plot. (b) Tetragonal case including $K_{m c}$ at $0 \mathrm{~K}$ : OP (blue), IP along [100] (red) and IP along [110] (green) curves; insert: $\mathrm{E}(\theta, \varphi)$.

The Ni cubic magnetocrystalline constant $K_{m c}$ can be neglected at $300 \mathrm{~K}\left(-0.05 \times 10^{5} \mathrm{~J} . \mathrm{m}^{-3}\right)$ and increases significantly at $0 \mathrm{~K}\left(-1.2 \times 10^{5} \mathrm{~J}^{-3} \mathrm{~m}^{-3}\right)$ [27]. If we additionally take $K_{m c}$ into consideration, the anisotropy adopts a tetragonal symmetry. The anisotropy energy $E$ per unit volume $V$ as a function of the magnetization orientation $(\theta, \varphi)$ can be written as:

$$
\frac{E}{V}=-K_{1} \cos ^{2} \theta-K_{m c} \sin ^{4} \theta+\frac{K_{m c}}{4} \sin ^{4} \theta \sin ^{2}(2 \varphi)
$$

where $K_{1}=K_{m e}+K_{m s}+K_{m c}$. For the hard axis, the magnetocrystalline contribution induces a curvature in the magnetization slope and increases the saturation field determined by $\left|K_{1}\right|$. In the easy plane, the cycles are now open (Fig.5(b)). It should be underlined that the IP quadratic modulation of the anisotropy energy density is independent of the strain and equal to $\left|K_{m c}\right| / 4$. At low temperature, $\left|K_{m c}\right| / 4$ is around $0.3 \times 10^{5} \mathrm{J.m}^{-3}$, i.e. nonnegligible in comparison with $K_{m s}$ and $K_{m e}$. The opening of the IP cycles at $0 \mathrm{~K}$ varies from the minimal value along [100] $\left(\mu_{o} H_{c,[100]}=0.13 \mathrm{~T}\right)$ to the maximal one along [110] $\left(\mu_{o} H_{c,[110]}\right.$ $=0.49 \mathrm{~T})$. However, no difference was observed along the [100] or [110] direction in the samples (Fig.4). Furthermore, the IP coercive fields observed $\left(H_{c, \|}\right)$ are too large to be attributed to $K_{m c}$ (Tab.2). These features indicate that coercitive characteristics observed herein are not related to a magnetocrystalline contribution. In the following, we will neglect $K_{m c}$ and consider strain as the main effect.

It appeared clearly that the mean tensile strain $\left\langle\epsilon_{z z}-\epsilon_{r r}\right\rangle$ implies a mean negative value of $\left\langle K_{1}\right\rangle$ and determines the general anisotropy of the OP hard axis. However, a tensile strain even with a broad distribution does not give rise to any IP coercivity. In order to take into account local energy barriers in the easy 
plane, it is thus necessary to introduce a second uniaxial constant $K_{r}$. Furthermore, the second anisotropy axes should be randomly oriented in plane, yielding a locally anisotropic and overall isotropic behavior. It is highly plausible that the strain in the plane $\epsilon_{i j}(i, j=\mathrm{x}$ or $\mathrm{y})$ is not locally homogeneous along a NW. It is then possible to define a local IP anisotropy by its constant $K_{r}$ and axis orientation $\varphi_{o}$ (§Appendix A). One remarks that all IP anisotropies are covered by positive values for $K_{r}$ and in the range $[0, \pi]$ for $\varphi_{o}$. The local anisotropy is thus biaxial (Fig.6) and the energy density $E / V$ can be written as:

$$
\frac{E}{V}=-K_{1} \cos ^{2} \theta-\frac{K_{r}}{2} \sin ^{2} \theta \cos \left[2\left(\varphi-\varphi_{o}\right)\right]
$$

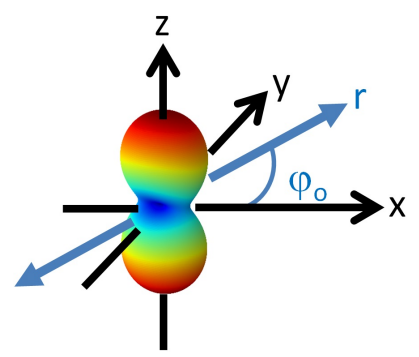

Figure 6: Plot of the local anisotropy energy surface $E(\theta, \varphi)$ with $K_{r} / K_{1}=0.5 /(-$ $1.5)$, where the easy axis is oriented in-plane at the azimuthal angle of $\varphi_{o}$.

It is likely that even a biaxial description with IP 2D randomly oriented anisotropies might be too simplistic to provide an exhaustive description of highly strained Ni NWs. Indeed, the latter does not explain the origin of the OP coercivity. If we consider all the strain components including the local axial shear ones $\epsilon_{i j}(i=$ (x or $\mathrm{y})$ and $j=\mathrm{z}$, and vice versa), the resulting magnetoelastic effect leads to a local hard axis oriented off the OP axis (\$Appendix A), which means that the hard axis or easy plane is wandering. Random ferromagnets with a wandering easy axis were described by Chudnovsky et al. [28]. Analysis of a wandering hard axis or easy plane has not been reported in the literature to our knowledge.

\subsubsection{Activation lengths according to the RMA model}

Considerations based on the SW coherent model rely on the anisotropy constants, independently of the magnetic activation volume. However, the activation volume is fundamental in magnetization switching. To describe local magnetization correlations, we take as the scale the width of a Bloch domain wall, $L_{o}=\pi \sqrt{A /|K|}$ where $A$ is the exchange stiffness constant. The exchange strength prevents the length of local magnetization correlations to be shorter than the width of one domain wall. For Ni, $L_{o}$ is equal to $29 \mathrm{~nm}$ for $|K|$ of $10^{5} \mathrm{~J} . \mathrm{m}^{-3}$. For our ultrathin NWs, the magnetization is thus always coherent in an inplane section of a NW, given the smallness of the present NW diameters with respect to $L_{o}$. On the other side, it was established that magnetization switching is in general localized along a NW, initiated by morphological, structural or anisotropy inhomogeneities and nucleated over the length of a domain wall [29-31].
It should be underlined that typical lengths of $L_{o}$ around 29 $\mathrm{nm}$ are larger than the structural coherence lengths ranging from 6 to $13 \mathrm{~nm}$. It results that random magnetic anisotropy (RMA) effects should be taken into account [28, 32]. According to Herzer [33, 34], the effective local anisotropy should be averaged or renormalized: the system finds equilibrium in a selfconsistent way with a local constant $\langle K\rangle$, which is averaged over $N$ neighboring domains, and a local magnetic correlation length $L=\pi \sqrt{A /|\langle K\rangle|}$. For the 3D case where $K$ and the size $L_{s}$ of the structural domains are fixed and only the anisotropy axis orientations random, the RMA links the structural and magnetic correlation lengths as follows: $L / L_{o}=\left(L_{o} / L_{s}\right)^{3}$, which shows that the magnetic correlation length increases with decreasing size of the domains. This allowed to successfully explain the coercivity reduction in soft magnetic nanocrystalline films [33$35]$. For the 1D case, a similar consideration leads to:

$$
L / L_{o}=\left(L_{o} / L_{s}\right)^{1 / 3}
$$

It results from the RMA model that the measured anisotropy constants are not directly those determined by the magnetoelastic and magnetostatic effects, but those further softened by local averaging. For instance, when taking structural domains of $L_{s}=6 \mathrm{~nm}$ length and a magnetic correlation length of $L_{o}=$ $29 \mathrm{~nm}$, about 8 structural domains of $L=49 \mathrm{~nm}$ total length act magnetically together during magnetization switching, i.e. as a macrospin. The transformation of the structurally determined $K$ to the magnetically measured $\langle K\rangle$ is complex and needs further thorough theoretical analysis, as it depends on the neighboring relationship between structural coherent domains along a NW. Here, we checked that the RMA model fits with the case of the present Ni NWs (§Appendix B).

\subsubsection{Simulation of the IP hysteresis cycles}

Based on the above considerations, we take a magnetically correlated domain as a macrospin with given anisotropy constants. By doing so, the local exchange effect between structural coherent domains is implicitly included according to the RMA model. Hysteresis cycles are then calculated, by adding the cycles from an anisotropy constant distribution representing the whole assembly of NWs. Differences between the modeling of an assembly of non-interacting macrospins and the experiments should then hint at lacking parameters: dipolar interactions between macrospins and exchange coupling between neighboring macrospins.

The SW hysteresis cycles, with a rectangular shape for an easy axis and linear relationship for a hard axis, are too idealized. Approaches to include thermal agitation have been proposed. For a hard axis, the thermal effect on the magnetization can be easily added, using Boltzmann distribution, $\propto \exp \left[-E /\left(k_{B} T\right)\right]$, where $E$ is the sum of the Zeeman and anisotropy energies and $k_{B}$ the Boltzmann constant. Along an easy axis, the thermal agitation should be considered, in addition to thermal activation. According to the Néel-Brown theory [36] and for a typical measurement duration of one second, $25 k_{B} T$ is taken as the thermal activation threshold against an energy barrier in the system. Here, we adopt the approach of 
Franco and Conde [37]: the magnetization remains in its local energy well and is Boltzmann distributed, as long as the height of the well is above $25 k_{B} T$. This procedure improves the shape of the calculated cycles, especially in vicinity of the coercive field.

As a consequence of thermal activation and according to Sharrock's law [38], the coercive field decreases as a function of the temperature as $T^{1 / 2}$. For real systems, an exponent of $2 / 3$ has often been found $[29,39,40]$. When adding the cycles from a given anisotropy distribution, we apply the thermal evolution with the exponent of $2 / 3$ for each subjacent cycle. When one cycle is closed, reaching its blocking temperature, a Langevin behavior is applied.
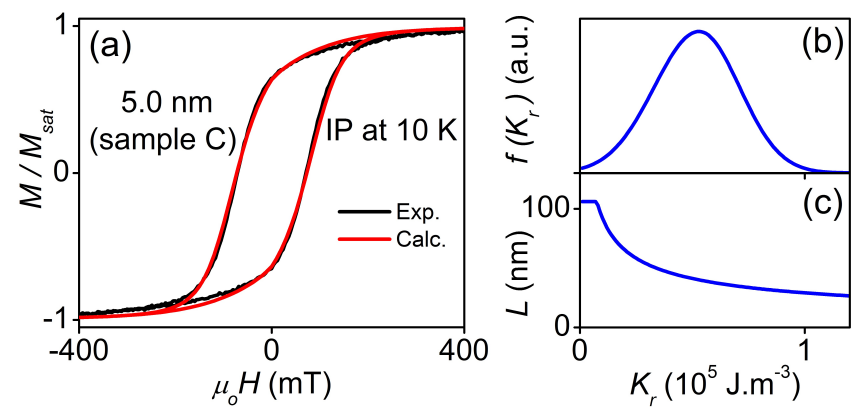

Figure 7: IP hysteresis behavior at $10 \mathrm{~K}$ in sample C. (a) Measured and calculated IP cycles. (b) Distribution of the radial anisotropy constant $f\left(K_{r}\right)$ used in the calculation. (c) Length $L$ of $\pi \sqrt{A / K_{r}}$, limited by the physical NW length of $106 \mathrm{~nm}$ for this sample.

The model for the easy plane is based on an in-plane randomly oriented uniaxial anisotropy with the constant $K_{r}$, irrespective of the negative value of $K_{1}$ for the hard axis. The IP hysteresis cycles are calculated considering a distribution $f\left(K_{r}\right)$ and associating a length $L$ equal to $\pi \sqrt{A / K_{r}}$ to each value of $K_{r}$. Figure 7 displays the case of sample $\mathrm{C}$ at $10 \mathrm{~K}$. The anisotropy distribution $f\left(K_{r}\right)$ used (Fig.7(b)) has the mean

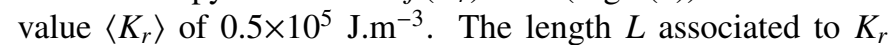
(Fig.7(c)) increases from $33 \mathrm{~nm}$ for "hard" parts $\left(K_{r} \sim 0.8 \times 10^{5}\right.$ J.m ${ }^{-3}$ ) to the NW physical length of $106 \mathrm{~nm}$ for "soft" parts $\left(K_{r} \leq 0.08 \times 10^{5} \mathrm{~J} . \mathrm{m}^{-3}\right)$, while the mean length $\langle L\rangle$ is $43 \mathrm{~nm}$.

Surprisingly, such a simplified model with non-interacting macrospins fits quite well with the experiments. Only small deviations upon approaching saturation are observed. The model fits also with the cycles at low temperature in samples A and B. It suggests that remaining interactions between the macrospins in the NWs are small. Additional evidence for the accuracy of our 2D random model for the easy plane is also provided by an analysis of the remanence $m_{R, \|}$, which is found equal to 0.61 at $T=10 \mathrm{~K}$ for sample $\mathrm{C}$ for instance. It should be recalled that $m_{R}$ is equal to 0.50 for a 3D random assembly and 0.64 for a $2 \mathrm{D}$ one.

\subsubsection{Behavior along the hard axis}

IP local energy barriers analyzed until now do not give rise to the OP coercivity. As discussed previously, a wandering of the hard axis (Fig.8) leads to the opening of the OP cycles. This means that the local axial shear strains should be taken into account when considering the magnetoelastic effect. The problem involves multiple parameters and their distributions over the NW assembly: $K_{r}, K_{1}$, the wandering angle $\theta_{o}$ and the azimuthal angle $\phi_{o}$ of the easy axis IP projection. Fitting of the OP measured cycles has not been performed, because of the large number of adjustable parameters.
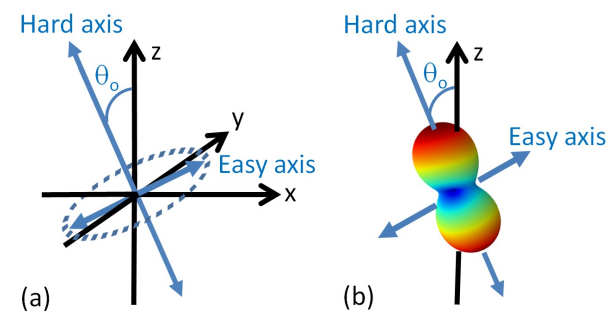

Figure 8: Sketch of local anisotropy energy surface $E(\theta, \varphi)$ where the hard axis is tilted with respect to the $\mathrm{z}$ axis by an angle of $\theta_{0}$. (a) Definition of the axes where the dashed cycle represents possible easy axes; (b) plot of $E(\theta, \varphi)$.

Table 3: Mean anisotropy values at $10 \mathrm{~K}$ of samples A-C: $\left\langle K_{r}\right\rangle$ deduced from the fitting of the measured IP cycles; $\left\langle K_{1}\right\rangle$ and the mean wandering angle $\left\langle\theta_{o}\right\rangle$ estimated from the value of $\left\langle K_{r}\right\rangle$, the OP saturation field and the OP remanence.

\begin{tabular}{cccc}
\hline Sample & $\begin{array}{c}\left\langle K_{r}\right\rangle \\
\left(10^{5} \mathrm{~J} \mathrm{~m}^{-3}\right)\end{array}$ & $\begin{array}{c}\left\langle K_{1}\right\rangle \\
\left(10^{5} \mathrm{~J} . \mathrm{m}^{-3}\right)\end{array}$ & $\begin{array}{c}\left\langle\theta_{o}\right\rangle \\
\left({ }^{\circ}\right)\end{array}$ \\
\hline A & 0.27 & -1.8 & 8 \\
B & 0.41 & -1.8 & 21 \\
C & 0.51 & -1.2 & 24 \\
\hline
\end{tabular}

The mean values of $K_{1}$ and $\theta_{o}$ can be approximately evaluated by using the OP remanence $m_{R, \perp}$ and saturation field $H_{s, \perp}$. Within the SW model, the remanence along the OP axis is of the order of $\sin \left(\theta_{o}\right)$. The OP saturation field is given by the difference $\Delta K$ of the anisotropy constants between the hard and easy axes: $\mu_{o} H_{s, \perp}=2 \Delta K / M_{s}$ and $\Delta K \sim\left|K_{1}-K_{r} / 2\right| / \cos \left(2 \theta_{\mathrm{o}}\right)$. From the measured values (Tab.2), the mean values of $K_{1}$ and $\theta_{o}$ are listed in Table 3, together with that of $K_{r}$ previously determined by the fitting of the IP cycles. The strain effect is clearly reflected in $\left\langle K_{1}\right\rangle$ and $\left\langle\theta_{o}\right\rangle$. With decreasing axial tensile strain from sample A to sample $\mathrm{C}$, the absolute value of $\left\langle K_{1}\right\rangle$ is decreased, while the wandering angle $\left\langle\theta_{o}\right\rangle$ increases from 8 to $24^{\circ}$. It should be noticed that the absolute values of $\left\langle K_{1}\right\rangle$ are lower by $22-47 \%$ in comparison to those calculated from the mean strain and the shape effect, for the three samples. The lowering of $\left\langle K_{1}\right\rangle$ should be related to the RMA local averaging.

\subsection{Thermal evolution of the anisotropy}

\subsubsection{Behavior in the easy plane}

The typical thermal evolution of the coercivity is illustrated in Figure 9 (sample A in IP configuration). The striking point here is the evolution of $H_{c}$ as a function of the temperature (Fig.9(b)). Along the easy plane or the hard axis, the decay of $H_{c}(T)$ is exponential for all measured samples including those 

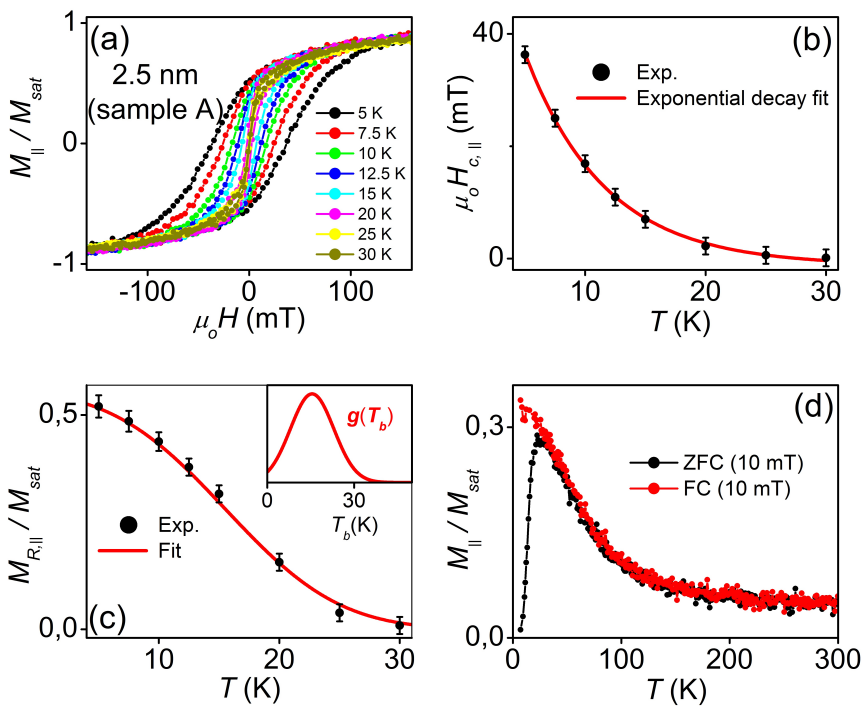

Figure 9: IP thermal evolution of the coercivity in sample A. (a) IP hysteresis cycles from 5 to $30 \mathrm{~K}$. (b) Coercive fields $\mu_{o} H_{c, \|}$ extracted from the cycles and exhibiting an exponential decay with increasing temperature. (c) Remanence extracted from the cycles and fitted by the partition function of a volume distribution of $T_{b}: M_{R, \|} / M_{s a t} \propto \int_{T}^{+\infty} g\left(T_{b}\right) d T_{b}$. Insert: corresponding distribution $g\left(T_{b}\right)$. (d) IP ZFC/FC measurements made with a bias field of $10 \mathrm{mT}$.

not shown here. This is a clear departure from the power law predicted by Sharrock [38].

The blocking temperature $T_{b}$ is the maximal value above which the coercivity disappears, as shown by the closure of the cycles (Fig.9(a)) or the convergence of ZFC/FC curves (Fig.9(d)). The remanence as a function of the temperature reflects a broad distribution of blocking temperatures in the NWs (Fig.9(b)). As a first estimate, the remanence could be understood as the sum of the magnetization of all parts still blocked at a given temperature in the SW model: $\propto \int_{T}^{+\infty} g\left(T_{b}\right) d T_{b}$ where $g\left(T_{b}\right)$ the volume distribution of $T_{b}$. For sample A with the maximum value of $T_{b}$ around $26 \mathrm{~K}$, this yields a mean value $\left\langle T_{b}\right\rangle$ of $15.5 \mathrm{~K}$ and a variance $\sigma\left(T_{b}\right)$ of $7.5 \mathrm{~K}$ (Inset in Fig.9(c)). Broad distributions of $T_{b}$ were similarly found in all the samples along the NWs and in the plane.

The blocking temperature $T_{b}$ increases with increasing NW diameter, or approximately linearly with increasing NW section area. This is a robust relationship verified also in other samples not shown here. $T_{b}$ is proportional to the energy barrier in the system which can be expressed by $K_{e f f} V^{*}$, where $V^{*}$ is the activation volume and $K_{\text {eff }}$ the associated effective anisotropy constant. For NWs, $V^{*}=L S$, with $L$ the activation length and $S$ the NW section area. A $T_{b}$ scaling with $S$ would mean that the product $K_{e f f} L$ is similar in all cases. This observation indicates that the activation does not concern the whole physical length of a NW, contrary to the SW model, but a length of a NW correlated with the effective anisotropy constant.

\subsubsection{Coercive field distribution in the easy plane}

In order to detail the thermal evolution of the coercivity in the easy plane, the anisotropy constant $K_{r}$ and the activation length require closer analysis. If we take a distribution $f\left(K_{r}\right)$, such as in Fig.7(b) for sample C, and apply the thermal activation according to Sharrock's law, the exponentially decreasing $H_{c}(T)$ as a function of the temperature cannot be reproduced. It was claimed that different shapes of $H_{c}(T)$ could be retrieved by changing the anisotropy distribution [41]. For the present case, we have not found a suitable anisotropy distribution that could lead to an exponentially decreasing $H_{c}(T)$ for all the samples and in both IP and OP configurations. Instead of a static, i.e. temperature independent distribution $f\left(K_{r}\right)$, our modeling suggests that the anisotropy distribution narrows and peaks at lower values with increasing temperature, which implies that $H_{c}(T)$ decreases more pronouncedly than suggested by Sharrock's law. In other terms, an additional thermally activated mechanism is at work in the NWs.
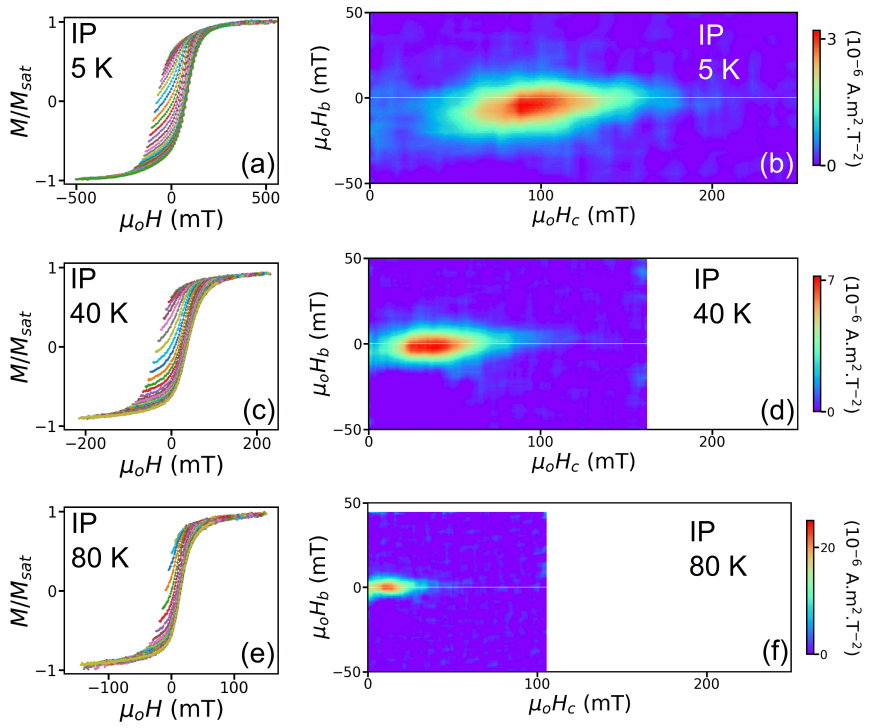

Figure 10: IP FORCs of sample C. (a) Reversal curves measured at $5 \mathrm{~K}$ after saturation at $800 \mathrm{mT}$ prior to each reversal. (b) FORC map at $5 \mathrm{~K}$. (c) Reversal curves measured at $40 \mathrm{~K}$ after saturation at $500 \mathrm{mT}$ (every third measured curve is plotted). (d) FORC map at $40 \mathrm{~K}$. (e) Reversal curves measured at $80 \mathrm{~K}$ after saturation at $300 \mathrm{mT}$ (every fifth measured curve is plotted).

In order to gather additional information on such a mechanism, FORC measurements were performed at 5, 40 and 80 $\mathrm{K}$ in sample $\mathrm{C}$ (Fig.10). FORC measurements allowed to retrieve the coercive field distributions $\rho\left(H_{c}\right)$ at the three temperatures, by integrating the FORC intensities on the $\mu_{o} H_{c}$ axis. In the next section $(\$ 3.3 .3)$, we will examine whether the thermal evolution of $\rho\left(H_{c}\right)$ differs from Sharrock's law, then check the temperature dependency of the anisotropy distribution.

The FORC measurements provide also information on the bias fields $H_{b}$, reflecting the interaction between NWs. This is especially visible at $5 \mathrm{~K}$ where the intensity is distributed with a slight slope below the $\mu_{o} H_{c}$ axis, i.e. the values of $H_{b}$ are negative or subjacent hysteresis cycles biased towards negative fields due to the mutual interaction. It should be pointed out that in easy-axis NW arrays, the bias fields are positive [42, 43]. In the mean-field framework [20, 44, 45], the interaction field $H_{i n t}$ is proportional to the magnetization den- 
sity: $H_{\text {int }}=k M / V=k P M_{s} m$, where $k$ is a constant, $m$ the reduced magnetization, $P$ the film porosity and $V$ the film volume. In the case of dipolar interactions, the demagnetizing field felt by a NW from the film mean magnetization density leads to $k=+1 / 2$ for the easy-plane case, instead of $k=-1$ for the easy-axis case [46, 47]. The bias field is expected to be smaller than $k P M_{s}(30 \mathrm{mT})$, as observed in the FORC maps. We checked that the bias field $H_{b}$ in the map at $5 \mathrm{~K}$ can be entirely substracted when replacing the applied field and reversal field by the effective local fields: $H_{\text {eff }}=H+H_{\text {int }}$ with $k=+1 / 2$. This leads to a small correction to $\rho\left(H_{c}\right)$. For simplification, we do not take into account this last correction, especially since the bias effect becomes negligible at 40 and 80 K.

\subsubsection{Analysis of the IP thermal evolution of anisotropy}

IP hysteresis cycles $M(H)$ in sample $\mathrm{C}$ were simulated by assuming a temperature-dependent anisotropy distribution, chosen such that the calculated cycles and coercive fields $H_{c}$ fit the measured data (Fig.11(a-c)). With this procedure, the volume distribution of anisotropy $\rho\left(K_{r}\right)$ was determined at different temperatures: $\rho\left(K_{r}\right) \propto f\left(K_{r}\right) \times L\left(K_{r}\right) \times S$ with $S$ the NW area. Figure $11(\mathrm{~d})$ shows $\rho\left(K_{r}\right)$ obtained at 5, 40 and $80 \mathrm{~K}$ and illustrates the shift and narrowing of the distribution $\rho\left(K_{r}\right)$ towards smaller values with increasing temperature, i.e. a thermal softening of anisotropy. Sharrock's thermal reduction of coercivity is expressed by the relationship between $H_{c}$ and $K_{r}$ for a 2D random assembly:

$$
\mu_{o} H_{c}=0.51 \times \frac{2 K_{r}}{M_{s}} \times\left[1-\left(\frac{25 k_{B} T}{K_{r} L S}\right)^{2 / 3}\right]
$$

for $25 k_{B} T<K_{r} L S$. Sharrock's effect can be directly visualized by comparison of Figures 11(d) and 11(e), i.e. the transformation of $\rho\left(K_{r}\right)$ to $\rho\left(H_{c}\right)$ according to Eq.4. A remarkable agreement is observed between the calculated and FORC measured coercive field distributions $\rho\left(H_{c}\right)$. This validates the fact that the exponential reduction of $H_{c}(T)$ in temperature is due to two effects: thermally induced changes of the anisotropy distribution $\rho\left(K_{r}\right)$ and Sharrock's thermal reduction of coercivity.

The calculation was performed for all measured IP $M(H)$ cycles and the aforementioned tendency confirmed in the samples. It is instructive to analyze $\left\langle K_{r}\right\rangle$ and $\langle L\rangle$ with respect to the dimensionless ratio $T / T_{b}$ (Fig.12). We would like to emphasize the values extrapolated at $0 \mathrm{~K}$ and the temperature dependence of $\left\langle K_{r}\right\rangle$ and $\langle L\rangle$. The mean values $\left\langle K_{r}\right\rangle$ at $0 \mathrm{~K}$ are close for

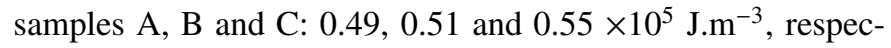
tively. From these values, we estimate an inhomogeneity in radial strain ranged between $0.4 \%$ and $0.5 \%$, to be compared with the mean radial strain $\left\langle\epsilon_{r r}\right\rangle$ of $-0.3 \%$ to $-1.0 \%$ measured in the samples (Tab.1). Correlatively, the mean lengths $\langle L\rangle$ at $0 \mathrm{~K}$ are similar: 45, 43 and $42 \mathrm{~nm}$ are calculated for samples A, B and $\mathrm{C}$, respectively. This indicates that irrespective of the details of the IP strain state in NWs, local RMA averaging leads to similar magnetic correlation lengths. With increasing $T / T_{b}$ until the blocking temperature, the reduction of $\left\langle K_{r}\right\rangle$ reaches $46-85 \%$
(Fig.12(a)), while the mean magnetic correlation length $\langle L\rangle$ increase (Fig.12(b)). The evolution of $\left\langle K_{r}\right\rangle$ and $\langle L\rangle$ as a function of $T / T_{b}$ follows a general and similar tendency in the samples, despite the fact that $T_{b}$ varies significantly from one sample to another.
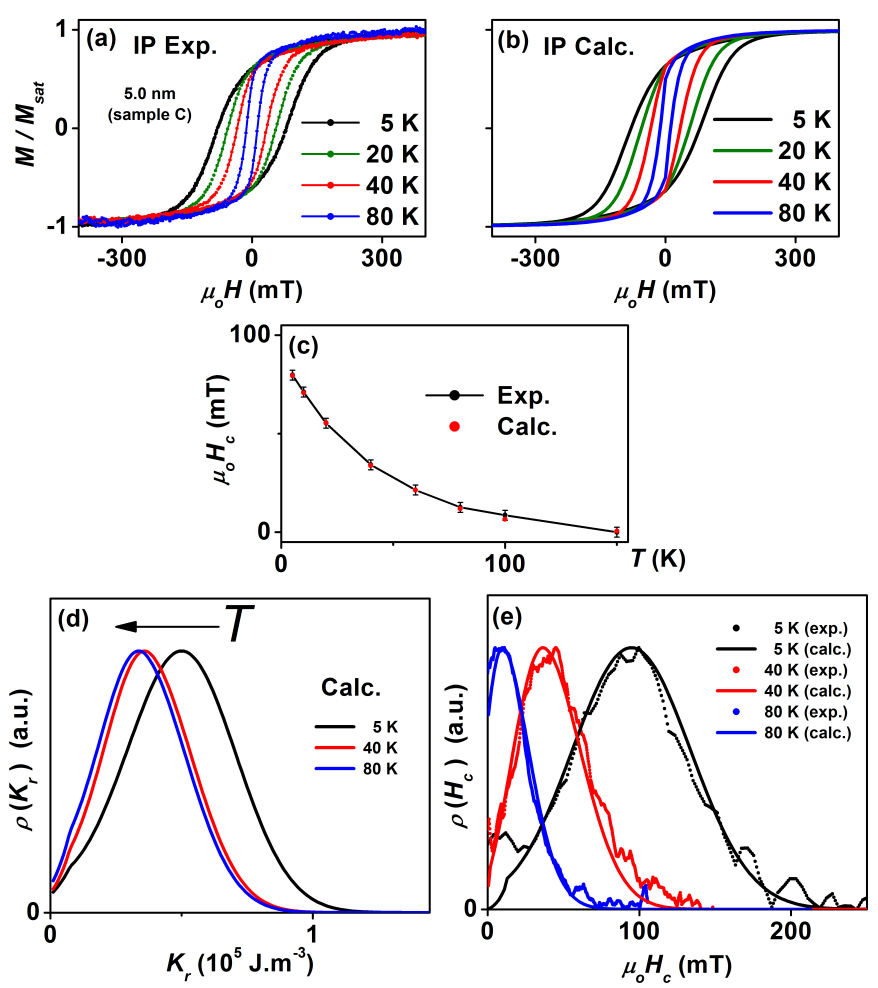

Figure 11: IP thermal evolution of the coercivity in sample C. (a) Selected cycles $M(H)$ measured at 5, 20, 40 and $80 \mathrm{~K}$. (b) Selected cycles at 5, 20, 40 and $80 \mathrm{~K}$ calculated with an anisotropy distribution which is adjusted as a function of temperature in such a way that the calculated coercive fields $H_{c}$ fit the measured ones (c). (d) Volume distributions of anisotropy $\rho\left(K_{r}\right)$ at 5 , 40 and $80 \mathrm{~K}$ deduced from the calculation: the arrow illustrates the shift of $\rho\left(K_{r}\right)$ towards smaller values with increasing temperature. (e) Coercive field distribution $\rho\left(H_{c}\right)$ deduced from $\rho\left(K_{r}\right)$ by applying Sharrock's equation at 5, 40 and $80 \mathrm{~K}$ and comparison with $\rho\left(H_{c}\right)$ extracted from the FORC measurements.
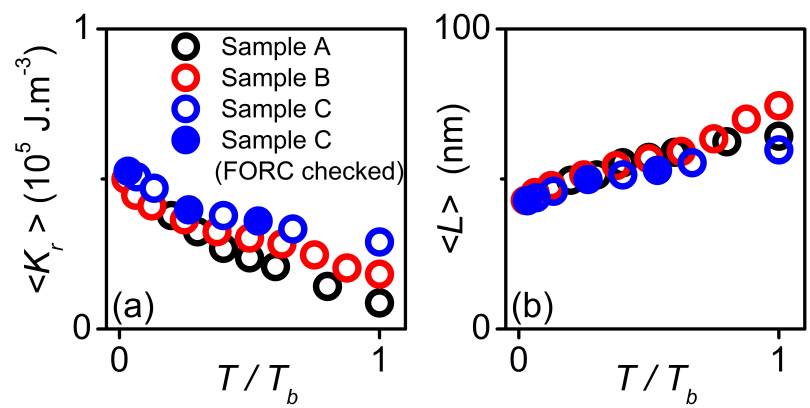

Figure 12: IP thermal evolution of (a) the mean anisotropy constant $\left\langle K_{r}\right\rangle$ and (b) the magnetic lengths $\langle L\rangle$, deduced from the fitting of all measured IP cycles $\mathrm{M}(\mathrm{H})$ in samples $\mathrm{A}, \mathrm{B}$ and $\mathrm{C}$. The three values validated by the FORC measurements in sample $\mathrm{C}$ are marked by blue solid circles in (a) and (b). 


\section{Discussion}

\subsection{Thermal softening of anisotropy}

The unusual thermal softening of anisotropy constitutes a key feature of the present NWs. Many studies reported on variations of anisotropy with temperature, related to a variation of physical constants, such as magnetocrystalline constants [48]. In the present work, the samples show a similar shift of the anisotropy distribution, over different temperature ranges until $150 \mathrm{~K}$. This suggests a general thermally activated process and not the thermal variation of a constant that would impact the samples differently as a function of temperature. Intrinsic and extrinsic mechanisms should be considered in order to understand the thermal softening of anisotropy. As the magnetic correlation lengths and the anisotropy constants are fully equivalent within the RMA model, a thermally induced increase of the magnetic length implies a reduction of the anisotropy constant and vice versa.

One basic intrinsic mechanism that can be considered is the thermal increase of the domain wall (DW) width $L_{o}(T)$. It can be shown that the DW width increases linearly with temperature (§Appendix B). This effect is important for the understanding of the DW motion in nanostructures [49, 50]. It is a general phenomenon for DWs, including ferroelectric ones [51]. For sample C, the mean DW width $L_{o}(T)$ is estimated to $31 \mathrm{~nm}$ at 0 $\mathrm{K}$ and increases by $6 \%$ at $T_{b}$. According to the RMA model, the increase of $L_{o}(T)$ induces consequently that of the local effective $L(T)$. This can be estimated using the scaling relationship in Eq.(3) which leads to: $L(T) / L(0)=\left[L_{o}(T) / L_{o}(0)\right]^{4 / 3}$. Thus, the total intrinsic thermal increase of $L(T)$ from $0 \mathrm{~K}$ to $T_{b}$ is evaluated to lie around $8 \%$ in the present case (§Appendix B). The linear thermal increase of $L(\mathrm{~T})$ fits with an exponential decay of $H_{c}(T)$, but the increase estimate of $8 \%$ of $L(T)$ until $T_{b}$ is still far from $44 \%$ observed (Fig.12(b)) and does not cover the decay rate of $H_{c}(T)$.

An exponential decay of $H_{c}(T)$ has already been observed in other types of random nanomagnets and composites [52-54] and this behavior has been attributed to the pinning of DWs, suggesting that DW pinning might be inherent to nanostructured magnets and the thermal softening of anisotropy a general feature of RMA. Intrinsic DW pinning at lattice planes occurs for narrow DWs and can be ruled out for the present large DWs. Extrinsic pinning due to defects can be envisioned, but a description of this phenomenon requires an analysis going beyond a phenomenological description of DW pinning [55]. Kirby et al. proposed a mechanism of incoherent magnetization reversal in thin films with a $1 / H$ field dependence of energy barriers [56]. This model predicts that the activation volume varies linearly with $T$ at low temperature and as $T^{2}$ at high temperature.

This quick review indicates that the thermal softening of anisotropy observed in the present NWs might be qualitatively related to intrinsic and/or extrinsic thermal activated process. Further theoretical work will be needed to establish a quantitative analysis, particularly with regard to possible defects responsible for DW pinning in vertically epitaxied NWs.

\subsection{Sources of anisotropy}

In the present paper, we focused on the magnetoelastic effect due to the epitaxial strain experienced by Ni NWs epitaxied in a STO matrix. To complement our analysis, other possible causes of strain and anisotropy should be examined. Firstly, it is intriguing that the magnetocrystalline effect is not observable in the measurements, although $\left|K_{m c}\right| / 4$ is of the same order of magnitude as $K_{r}$ at low temperature. Jorritsma and Mydosh [57] reported Ni NWs horizontally aligned and strained on patterned InP substrate where $K_{m c}$ was also ignored for the whole range of 5-300 K. Based on broad strain distributions measured by XRD in our case, it is plausible that the strain distortion in the NWs destroys locally the tetragonal symmetry and then masks the $K_{m c}$ effect.

Surface anisotropy [58] becomes important for nanosized objects. The energy density induced scales as the inverse of the NW diameter. In the present case, we find no evidence for surface anisotropy contributions. Contrary to the case of a film, the cylindrical interface between the NWs and the matrix should make the contribution of surface anisotropy to coercivity negligible. Indeed, a uniform radial contribution does not contribute to $K_{r}$ as a consequence of IP inhomogeneity. A surface contribution is at least of second order: only the IP angular fluctuation of surface anisotropy contributes to the IP coercivity.

A possible thermal origin of the strain might also be envisioned. Indeed, the difference in the thermal expansion of the Ni NWs and matrix may constitute a source of strain, as demonstrated in Ni NWs electrodeposited on porous alumina templates $[59,60]$. The thermal expansion coefficient is 13.4 for $\mathrm{Ni}$ and around 9.5 for STO in $10^{-6} / \mathrm{K}$ units. From the growth temperature to the room temperature, the NWs would sustain a radial dilatation from the matrix and consequently a NW axial compression, contrary to the NW axial tensile strain observed. The maximal thermal strain can be estimated as the product of the temperature difference and the difference of the thermal expansion coefficients of $\mathrm{Ni}$ and STO. The maximal thermal strain is then $\leq 0.25 \%$, one order of magnitude lower than the measured strain.

It should be pointed out that absence of any coercivity is a specificity of the mean anisotropy with a hard axis and an easy plane (Fig.5(a)), contrasting with the case of an easy axis and a hard plane. Any additional contribution of cylindrical symmetry does not give rise to coercivity. This means that other sources of anisotropy, such as surface anisotropy or thermal strain, do not change the anisotropy, but only modify the hardness of the hard axis, i.e. the value of $K_{1}$. The magnetoelastic effect resulting from vertical epitaxial strain analyzed in this paper appears to be the dominating effect.

\section{Conclusion}

The mean anisotropy of the Ni NWs assembly, i.e. the OP hard axis and the mean isotropic IP easy plane, is induced by the mean axial tensile strain. The IP coercivity is induced by inplane strain inhomogeneities. Similarly, the appearence of an OP coercivity is interpreted by the wandering of the local hard 
axis caused by fluctuations of the local axial shear strains. The IP hysteresis cycles were simulated by macrospins of length scaling with the local RMA magnetic correlation length. The exponential decay of $H_{c}(\mathrm{~T})$ with temperature is related to Sharrock's effect and an additional thermally activated process of anisotropy softening.

The possibility of strain induced magnetic anisotropy reversal in NWs offers interesting perspectives to modulate their properties along the NW axis. However, strain fluctuations increase with the mean epitaxy strain. The strain fluctuations at the nanoscale lead to a softening of anisotropy as a result of local averaging and probably causes the additional thermal softening evidenced in this study. When heading for a maximum increase of anisotropy, a compromise should be found between average tensile strain and strain fluctuations. In the case of Ni NWs, this would correspond to a tensile strain much larger than $0.8 \%$ to overcome the shape effect and a low strain fluctuation over a typical magnetic correlation length of about $40 \mathrm{~nm}$. In this perspective, epitaxy of Ni NWs in a matrix with a vertical mismatch of around $3 \%$ with $\mathrm{Ni}$ would be interesting. Our analysis shows that the magnetic behavior of the Ni NWs ensemble can be grasped by using macrospins. The size of macrospins scales with the magnetic correlation length. The results obtained call for further thorough experimental and theoretical work in order to achieve an improved understanding of the anisotropy softening mechanisms at play in ultrathin NWs: a) local averaging of the magnetic anisotropy; b) intrinsic and/or extrinsic thermal softening of anisotropy.

\section{Acknowledgments}

X.W. gratefully acknowledges financial support of the Chinese Government Scholarship from China Scholarship Council (CSC No. 201608070062). M.H. acknowledges financial support from the French Embassy in Berlin (Service pour la Science et la Technologie) and Campus France. The authors gratefully thank S. Chenot for his assistance during PLD setup installation, F. Breton for his assistance and design of the PLD control system, S. Hidki and N. Casaretto for XRD measurements. The authors also thank IMPMC, CNRS-Sorbonne University, for access to the TEM facilities and Dr. J.-M. Guigner for his help. This work has been funded in part by the LabEx Matisse (French projects ANR-11-IDEX-0004-02/10-LABX0067-MATISSE).

\section{Appendix A. Local strain field and energy barriers}

The magnetoelastic and magnetostatic effects for a NW can be described by using an anisotropy constant tensor $\widehat{K}$ of second rank. The anisotropy energy density $E / V$ is written as: $E / V=-\mathbf{m} \cdot \widehat{K} \mathbf{m}$ with $\mathbf{m}$ the magnetization unit vector. When considering only the mean axial and radial strains, the only non-zero element of $\widehat{K}$ is: $K_{1} \equiv K_{z z}=-\mathrm{B}_{1}\left(\left\langle\epsilon_{z z}\right\rangle-\left\langle\epsilon_{r r}\right\rangle\right)+K_{m s}$, where the magnetoelastic coupling constant $B_{1}$ is equal to $-(3 / 2) \lambda_{001}\left(c_{11}-c_{12}\right)$.
The mean anisotropy does not give rise to any IP coercivity. A second IP uniaxial constant $K_{r}$ is used in order to account for local IP energy barriers. Furthermore, the anisotropy axes are IP randomly oriented. With this approach, the system becomes locally anisotropic and overall isotropic, in accordance with the experiments. $K_{r}$ expresses the fact that the in-plane strain $\epsilon_{i j}(i, j=x$ or $y)$ is not homogeneous along a NW. When the minimal radial strain is $\epsilon_{x x}$ and the maximum one $\epsilon_{y y}$, the constant $K_{r}$ induced by the magnetoelastic effect is: $K_{r} \equiv K_{x x}-K_{y y}=-B_{1}\left(\epsilon_{x x}-\epsilon_{y y}\right)$. A similar relationship is obtained for any IP orientation of the principal axes of $\epsilon_{i j}$ $(i, j=x$ or $y)$. In including the IP shear strain $\epsilon_{x y}$ with the associated magnetoelastic coupling constant $B_{2}\left(=-3 \lambda_{111} \mathrm{c}_{44}\right)$, the easy axis is oriented at the azimuthal angle of $\varphi_{o}$ and approximately aligned with one of the principal axes of $\epsilon_{i j}$. This is due to the fact that $B_{1}$ and $B_{2}$ of $\mathrm{Ni}$ are close: $B_{1}=9.38$ and $B_{2}=10$ in $10^{6} \mathrm{~J}^{-\mathrm{m}^{-3}}$ [61]. The corresponding anisotropy energy is given in Eq.(2). Depending on the values of $K_{1}$ and $K_{r}$, the landscape of the anisotropy energy of a biaxial system can become rather complex. According to the XRD results, $\mathrm{Ni}$ NWs are characterized by a broad distribution of $K_{1}$ centered on negative $K_{1}$ values. It is expected that the OP axis acts as a magnetization hard axis. $K_{r}$ values should be smaller than that of $K_{1}$, considering the weak values of $\mu_{o} H_{c, \|}$ observed. A typical case with $K_{1}<0$ and $K_{r}>0$ for the present NWs is illustrated in Figure 6. For IP configurations and regardless of the negative value of $K_{1}$, the mean magnetization is considered to remain in the plane.

The previous picture is incomplete, in case of high local strain distortion. When considering all strain components including the axial shear ones $\epsilon_{i j}(i=(r$ or $\theta)$ and $j=z)$, the tensor $\widehat{K}$ is written as:

$$
\widehat{K}=\left(\begin{array}{ccc}
K_{r} / 2 & 0 & K_{r z} \\
0 & -K_{r} / 2 & K_{\theta z} \\
K_{r z} & K_{\theta z} & K_{1}
\end{array}\right)
$$

where $K_{r z}=-B_{2} \epsilon_{r z}$ and $K_{\theta z}=-B_{2} \epsilon_{\theta z}$. The diagonalization of $\widehat{K}$ implies a local hard axis different from the OP axis. In other terms, the hard axis is wandering around the OP axis, as sketched in Figure 8.

The wandering angle $\theta_{o}$ depends on the ratio between the local axial shear strain and the tensile one. It follows that the magnetoelastic effect becomes more complex with increasing local strain distortion: a) the mean strain explains the existence of the hard axis and easy plane, without any coercivity; b) a biaxial anisotropy includes the local IP energy barriers; c) for real cases, a full 3D description of the strain field is needed. For highly strained Ni NWs, we show in this paper that the IP behavior can be described by an IP 2D randomly oriented local anisotropy and the OP one by including the wandering effect of the hard axis.

\section{Appendix B. Magnetic anisotropy softening}

Appendix B.1. IP local softening according to the RMA model

The RMA model is usually applied to 3D nanostructured materials [33, 34], when the size $L_{s}$ of structural domains becomes 
smaller than the magnetic correlation length $L_{o}$, where $L_{o}$ is taken to be equal to $\pi \sqrt{A / K}$ for the uniaxial case. When the anisotropy axis orientation is random, a magnetically correlated domain of volume $L^{3}$ includes $N$ structural domains of volume $L_{s}^{3}$, so that the anisotropy constant $\langle K\rangle$ averaged over the magnetic volume scales as $K / \sqrt{N}$ and the resulting magnetic length $L$ as $\pi \sqrt{A /\langle K\rangle}$.

The following specificities of the present NWs should be underlined: a) contrary to nanocrystalline films, nanostructuration here is induced by the strain; b) NWs are considered magnetically as $1 \mathrm{D}$ chains, so that $\mathrm{N}$ scales as $L / L_{s}$ instead of $\left(L / L_{s}\right)^{3}$ for $3 \mathrm{D}$; c) the anisotropy axis orientation is IP $2 \mathrm{D}$ random. Furthermore, $L_{s}$ is not uniform along a NW and ranges from 6 to $13 \mathrm{~nm}$, depending on the strain and the samples, as indicated by XRD measurements. Also, the anisotropy constant $K$ or equivalently $L_{o}$ is not unique and presents a distribution. However, a qualitative scaling consideration can be made with constant $L_{s}$ and $L_{o}$, similarly to the $3 \mathrm{D}$ case (Tab.B.4).

Table B.4: 3D and 1D RMA characteristics in terms of $L_{o} / L_{s}$, the ratio between the intrinsic magnetic length $L_{o}$ and the structural length $L_{S}: N$, number of structural domains within a magnetic volume; $L$, magnetic length from the RMA anisotropy constant $\langle K\rangle$ which is averaged over the magnetic volume.

\begin{tabular}{cccc}
\hline & $N$ & $L / L_{o}$ & $\langle K\rangle / \mathrm{K}$ \\
3D & $\left(L_{o} / L_{s}\right)^{12}$ & $\left(L_{o} / L_{s}\right)^{3}$ & $\left(L_{o} / L_{s}\right)^{-6}$ \\
1D & $\left(L_{o} / L_{s}\right)^{4 / 3}$ & $\left(L_{o} / L_{s}\right)^{1 / 3}$ & $\left(L_{o} / L_{s}\right)^{-2 / 3}$ \\
\hline
\end{tabular}

$N$ increases slowly with decreasing $L_{s}$ in $1 \mathrm{D}$. The averaged magnetic length $L$ scales as $L_{s}^{-1 / 3}$ (Eq.3), instead of $L_{s}^{-3}$ in $3 \mathrm{D}$. The reduction of the coercivity $(\propto\langle K\rangle)$ scales as $L_{s}^{6}$ in $3 \mathrm{D}$, as demonstrated in Ni nanocrystalline films [35], and as $L_{s}^{2 / 3}$ in 1D. It is to be noticed that the anisotropy energy of a magnetic domain is proportional to $\langle K\rangle L$ for the $1 \mathrm{D}$ case and scales as $L_{s}^{1 / 3}$. This means that fragmentation of the NWs into structural domains smaller than the magnetic length lowers the blocking temperature.

For sample $\mathrm{C}$ detailed in this study, the IP mean value of $\langle K\rangle\left(\equiv\left\langle K_{r}\right\rangle\right)$ measured at low temperature is about $0.55 \times 10^{5}$ $\mathrm{J} . \mathrm{m}^{-3}$ and implies a magnetic length $L$ of $42 \mathrm{~nm}$. Following the scaling rules in Table B. 4 and taking $L_{s}=13 \mathrm{~nm}$ (or $N \sim$ $3-4$, number of structural domains in a magnetic domain), the intrinsic magnetic length $L_{o}$ deduced is equal to $\sim 31 \mathrm{~nm}$ and the intrinsic anisotropy constant $K$ to $\sim 0.9 \times 10^{5} \mathrm{~J} . \mathrm{m}^{-3}$. This means that the anisotropy is reduced by $\langle K\rangle / K \sim 0.6$, due to the RMA local averaging for the present case.

\section{Appendix B.2. Intrinsic thermal softening}

The exchange energy of a DW of length $\mathcal{L}$ within a NW of area $S$ scales as $A(\alpha / \mathcal{L})^{2} \mathcal{L} S$ and the anisotropy energy as $(K / 2) \mathcal{L} S$, with $\alpha$ a constant. The total energy $E$ can thus be written as: $E(\mathcal{L})=\left[L_{o} / \mathcal{L}+\mathcal{L} / L_{o}\right] / 2 \times K L_{o} S$. Here, $\alpha$ is chosen equal to $\pi / \sqrt{2}$, so that $L_{o}$ fits with the usual Bloch DW width $\pi \sqrt{A / K}$. Minimization of the total energy allows to calculate the equilibrium DW length at $0 \mathrm{~K}$ which turns out to be $L_{o}$. At finite temperature, the mean DW width $L_{o}(T)$ is calculated by:
$L_{o}(T)=\left\langle\mathcal{L} \times \exp \left\{-E(\mathcal{L}) /\left[k_{B} T\right]\right\}\right\rangle$. The numerical resolution of the equation yields: $L_{o}(T) / L_{o} \approx 1+k_{B} T /\left[K L_{o} S\right] \times 1.534$. Taking the nanowire area in sample C, $S=19.6 \mathrm{~nm}^{2}$, and the values estimated previously, $K=0.9 \times 10^{5} \mathrm{~J} . \mathrm{m}^{-3}$ and $L_{o}=31$ $\mathrm{nm}$, the increase of $L_{o}(T)$ from $0 \mathrm{~K}$ to $T_{b}$ of $150 \mathrm{~K}$ is about $6 \%$. The RMA averaged magnetic length $L(T)$ scales as $\left[L_{o}(T)\right]^{4 / 3}$ (Tab.B.4) and increases consequently with $L_{o}(T)$. The total increase of $L(T)$ from $0 \mathrm{~K}$ to $T_{b}$ is estimated to be approximately equal to $8 \%$.

\section{Appendix B.3. Behavior along the hard axis}

For the 3D RMA model, the anisotropy tensor $\widehat{K}$ is traceless, so that the tensor describes the anisotropy fluctuation around zero [33, 34]. For the present case, the mean strain as well as $K_{1}$ is persistent and determines the mean hard axis and easy plane. The IP trace of the tensor $\widehat{K}$ in Eq.(A.1) is null, reflecting the IP anisotropy fluctuation. The mean values of $K_{r z}$ and $K_{\theta z}$ are also null, as a result of the local fluctuation of the axial shear strain.

For randomly distributed structural domains, $\left\langle K_{1}\right\rangle$ should remain stable against a distribution of domains in strain: $\left\langle K_{1}\right\rangle=$ $-B_{1}\left(\left\langle\epsilon_{z z}\right\rangle-\left\langle\epsilon_{r r}\right\rangle\right)+K_{m s}$. Only the variance $\sigma\left(K_{1}\right)$ should reduce as $1 / \sqrt{N}$ with the local averaging over $N$ domains. The estimated values of $\left\langle K_{1}\right\rangle$ listed in Table 3 do not follow this sketch and are lower than those calculated from the mean strain $\left(\left\langle\epsilon_{z z}\right\rangle-\left\langle\epsilon_{r r}\right\rangle\right)$ and the shape effect. This means that structural domains are not fully random. Indeed, XRD measurements suggest a correlation between neighboring domains, in the sense that less strained domains possess larger coherence lengths. This could explain the shift of $\left\langle K_{1}\right\rangle$ towards small values. It will be interesting and challenging to provide a full description of the anisotropy of the NWs by averaging locally the tensor $\widehat{K}$ from the strain field and considering not only the distribution of domains, but also the correlation between neighboring domains.

\section{References}

[1] L. Li, L. Sun, J.S. Gomez-Diaz, N.L. Hogan, P. Lu, F. Khatkhatay, W. Zhang, J. Jian, J. Huang, Q. Su, M. Fan, C. Jacob, J. Li, X. Zhang, Q. Jia, M. Sheldon, A. Alù, X. Li, H. Wang, Nano Lett. 16 (2016) 3936.

[2] A.V. Kabashin, P. Evans, S. Pastkovsky, W. Hendren, G.A. Wurtz, R. Atkinson, R. Pollard, V.A. Podolskiy, A.V. Zayats, Nat. Mater. 8 (2009) 867.

[3] J. Yao, Z. Liu, Y. Liu, Y. Wang, C. Sun, G. Bartal, A. M. Stacy, X. Zhang, Science 321 (2008) 930.

[4] S. Kawasaki, R. Takahashi, T. Yamamoto, M. Kobayashi, H. Kumigashira, J. Yoshinobu, F. Komori, A. Kudo, M. Lippmaa, Nat. Commun. 7 (2016) 11818.

[5] S. Lee, W. Zhang, F. Khatkhatay, H. Wang, Q. Jia, J.L. MacManusDriscoll, Nano Lett. 15 (2015) 7362.

[6] J. MacManus-Driscoll, A. Suwardi, A. Kursumovic, Z. Bi, C.-F. Tsai, H. Wang, Q. Jia, O.J. Lee, APL Materials 3 (2015) 062507.

[7] H. Wu, G. Chai, T. Zhou, Z. Zhang, T. Kitamura, H. Zhou, J. Appl. Phys. 115 (2014) 114105.

[8] X. Sun, J. Huang, J. Jian, M. Fan, H. Wang, Q. Li, J.L. MacManusDriscoll, P. Lu, X. Zhang, H. Wang, Mater. Horiz. 5 (2018) 536.

[9] L. Piraux, K. Renard, R. Guillemet, S. Mátéfi-Tempfli, M. MátéfiTempfli, V. Andrei Antohe, S. Fusil, K. Bouzehouane, V. Cros, Nano Lett. 7 (2007) 2563.

[10] S. Yang, H. Zhu, D. Yu, Z. Jin, S. Tang, Y. Du, J. Magn. Magn. Mater. 222 (2000) 97. 
[11] D. H. Qin, M. Lu, H. L. Li, Chem. Phys. Lett. 350 (2001) 51.

[12] A. Chen, J.M. Hu, P. Lu, T. Yang, W. Zhang, L. Li, T. Ahmed, E. Enriquez, M. Weigand, Q. Su, H. Wang, J.X. Zhu, J. L. MacManus-Driscoll, L.Q. Chen, D. Yarotski, Q. Jia, Sci. Adv. 2 (2016) e1600245.

[13] H. Zheng, J. Wang, S. E. Lofland, Z. Ma, L. Mohaddes-Ardabili, T. Zhao, L. Salamanca-Riba, S.R. Shinde, S.B. Ogale, F. Bai, D. Viehland, Y. Jia, D.G. Schlom, M. Wuttig, A. Roytburd, R. Ramesh, Science 303 (2004) 661.

[14] H.-J. Liu, Y.-Y. Liu, C.-Y. Tsai, S.-C. Liao, Y.-J. Chen, H-J. Lin, C.-H. Lai, W.-F. Hsieh, J.-Y. Li, C.-T. Chen, Q. He, Y.-H. Chu, Sci. Rep. 5 (2015) 12073

[15] V. Schuler, F.J. Bonilla, D. Demaille, A. Coati, A. Vlad, Y. Garreau, M Sauvage-Simkin, A. Novikova, E. Fonda, S. Hidki, V. Etgens, F. Vidal, Y. Zheng, Nano Research 8 (2015) 1964.

[16] X. Weng, M. Hennes, A. Coati, A. Vlad, Y. Garreau, M. Sauvage-Simkin, E. Fonda, G. Patriarche, D. Demaille, F. Vidal, Y. Zheng, Phys. Rev. Mater. 2 (2018) 106003

[17] F.J. Bonilla, A. Novikova, F. Vidal, Y. Zheng, E. Fonda, D. Demaille, V. Schuler, A. Coati, A. Vlad, Y. Garreau, M. Sauvage-Simkin, Y. Dumont, S. Hidki, V.H. Etgens, ACS Nano 7 (2013) 4022.

[18] M. Hennes, V. Schuler, X. Weng, J. Buchwald, D. Demaille, Y. Zheng, F. Vidal, Nanoscale 10 (2018) 7666

[19] V. Schuler, J. Milano, A. Coati, A. Vlad, M. Sauvage-Simkin, Y. Garreau, D. Demaille, S. Hidki, A. Novikova, E. Fonda, Y. Zheng, F. Vidal, Nanotechnology 27 (2016) 495601.

[20] C.R. Pike, A.P. Roberts, K.L. Verosub, J. Appl. Phys. 85 (1999) 6660.

[21] R.J. Harrison, J.M. Feinberg, Geochem. Geophys. Geosyst. 9 (2008) 1.

[22] K. Baberschke, Philos. Mag. 88 (2008) 2643.

[23] T. Burkert, O. Eriksson, P. James, S.I. Simak, B. Johansson, L. Nordstrom, Phys. Rev. B 69 (2004) 104426.

[24] E.C. Stoner, E.P. Wohlfarth, Trans. Roy. Soc. (London) A 240 (1948) 599

[25] C.A.F. Vaz, J.A.C. Bland, G. Lauhoff, Rep. Prog. Phys.71 (2008) 056501.

[26] R.C. Hall, J. Appl. Phys. 30 (1959) 816.

[27] R.R. Birss, G.J. Keeler, C.H. Shepherd, J. Phys. F: Met. Phys. 7 (1977) 1683.

[28] E.M. Chudnovsky, W.M. Saslow, R.A. Serota, Phys. Rev. B 33 (1986) 251.

[29] R. Skomski, H. Zeng, M. Zheng, D.J. Sellmyer, Phys. Rev. B 62 (2000) 3900

[30] M.I. Chipara, R. Skomski, D.J. Sellmyer, J. Magn. Magn. Mater. 249 (2002) 246

[31] F. Vidal, Y. Zheng, P. Schio, F.J. Bonilla, M. Barturen, J. Milano, D. Demaille, E. Fonda, A.J.A. de Oliveira, V.H. Etgens, Phys. Rev. Lett. 109 (2012) 117205

[32] R. Alben, J.J. Becker, M.C. Chi, J. Appl. Phys. 49 (1978) 1653

[33] G. Herzer, J. Magn. Magn. Mater. 294 (2005) 99.

[34] G. Herzer, The random anisotropy model, in: NATO Science Series II: Mathematics, Physics and Chemistry, Vol. 184, B. Idzikowski, P. Svec and M. Miglierini, Eds. (Kluwer Academic, Dordrecht, 2005) p. 15

[35] E. Kita, N. Tsukuhara, H. Sato, K. Ota, H. Yangaihara, H. Tanimoto, and N. Ikeda, Appl. Phys. Lett. 88, 152501 (2006)

[36] W.F. Brown, Jr., Phys. Rev. 130 (1963) 1677.

[37] V. Franco, A. Conde, J. Magn. Magn. Mater. 278 (2004) 28.

[38] M.P. Sharrock, J. Appl. Phys. 76 (1994) 6413.

[39] R. Skomski, J. Phys. Condens. Matter 15 (2003) R841.

[40] O. Fruchart, A. Thiaville, C. R. Physique 6 (2005) 921.

[41] W.C. Nunes, W.S.D. Folly, J.P. Sinnecker, M.A. Novak, Phys. Rev. B 70 (2004) 014419.

[42] C.R. Pike, A. Ross, T. Scalettar, G. Zimanyi, Phys. Rev. B71 (2005) 134407.

[43] C.-I. Dobrota, A. Stancu, J. Appl. Phys. 113 (2013) 043928.

[44] D.A. Gilbert, G.T. Zimanyi, R.K. Dumas, M. Winklhofer, A. Gomez, N. Eibagi, J.L. Vicent, K. Liu, Sci. Rep. 4 (2014) 4204.

[45] S. Ruta, Ondre Hovorka, P.-W. Huang, K. Wang, G. Ju, R. Chantrell, Sci. Rep. 7 (2017) 45218

[46] U. Netzelmann, J. Appl. Phys. 68 (1990) 1800.

[47] A. Encinas-Oropesa, M. Demand, L. Piraux, I. Huynen, U. Ebels, Phys. Rev. B63 (2001) 104415.

[48] Q. Bian, M. Niewczas, J. Appl. Phys. 117 (2015) 013909.

[49] N. Kazantseva, R. Wieser, U. Nowak, Phys. Rev. Lett. 94 (2005) 037206.

[50] J. Chico, C. Etz, L. Bergqvist, O. Eriksson, J. Fransson, A. Delin, A
Bergman, Phys. Rev. B 90 (2014) 014434.

[51] M. Foeth, P. Stadelmann, M. Robert, Physica A 373 (2007) 439

[52] J.I. Arnaudas, A. Del Moral, P.A.J. De Groot, J. Magn. Magn. Mater. 104-107 (1992) 115.

[53] K.M.B. Alves, L.C. Sampaio, A.P. Guimarfies, S.F. da Cunha, J Alloys Compd. 210 (1994) 325.

[54] X. Ning, Z.J. Wang, X. Zhao, C.-W. Shih, W.-C. Chang, Z. Zhang, IEEE Transactions on Magnetics 50 (2014) 1000304.

[55] A. Raghunathan, Y. Melikhov, J. E. Snyder, D. C. Jiles, IEEE Transactions on Magnetics 46 (2010) 1507.

[56] R.D. Kirby, M. Yu, D.J. Sellmyer, J. Appl. Phys. 87 (2000) 5696.

[57] J. Jorritsma, J. A. Mydosh, J. Appl. Phys. 84 (1998) 901.

[58] O. Hjortstam, K. Baberschke, J. M. Wills, B. Johansson, O. Eriksson, Phys. Rev. B55 (1997) 15026.

[59] S. Dubois, J. Colin, J.L. Duvail, L. Piraux, Phys. Rev. B61 (2000) 14315

[60] K.R. Pirota, E.L. Silva, D. Zanchet, D. Navas, M. Vázquez, M. Hernández-Vélez, M. Knobe, Phys. Rev. B76 (2007) 233410.

[61] D. Sander, Rep. Prog. Phys. 62 (1999) 809. 